\title{
New Age Recruitment Process - The AI Way
}

\author{
Mr G Anil Chandra Prasad
}

\author{
Research Scholar, \\ Department of Management, KL Deemed to be University
}

\begin{abstract}
Recruitment no doubt is the most challenging job for a HR professional, getting a Right Resource, in a Right Time, for a Right Position at a Right Cost is a perennially cumbersome assignment, and it is beyond identifying the right candidate, stimulating him to apply for the position in an organization. The toughest part of recruiting involves picking up the most suitable profile from huge pool of profiles, any small deviation in finding a right match not only reduces productivity but also brings down the team morale.

Picking the right profile rationally and within condensed time frames not only saves time but also reduces cost of recruitment, also scientific approach provides prolific results but this should not be mistaken with Taylorism and earlier Information Technology Tools and Portals. Artificial Intelligence (AI) is the novel means of recruitment which is serving as an efficient tool for screening profiles and enabling the recruiters to find the match with their respective job descriptions.
\end{abstract}

Yesteryears recruiters largely relied on several traditional sources, in the process they have generated data bank of applications, and manually sifting through it is next to impossible. Now comes the role of AI, which not only sifts the data but also analyses the same to identify the right match to the Job Descriptions they are looking for.

Thus AI tools have become essential prerequisites in enhancing recruitment efforts, this paper outlines the role of AI in leveraging recruitment process, and enlightens its significance in enhancing the efficiency of HR professionals in getting a right resource.

Keywords: Recruitment, Artificial Intelligence, Screening

\section{Introduction}

Results that organizations foresee are indisputably directly proportional to successful recruitments, recruiting right for the first time delivers both short term and long term rewards. Universal fact is that, it is not that simple to find the potential prospects and entice them. Recruitment in today's competitive environment demands for a strategic approach and has become one of the vital functional HR strategies.

Metamorphosis of recruitment process begun since the early 2000s. The Non-Conventional sources (viz., LinkedIn, Facebook, Telegram \&Twitter) didn't exist two decades ago. And this change can be attributed to swift changes in technologies

Recruiters job now has become relatively less complex with advent of recruitment software tools that are widely accessible and assist them in sourcing, screening and shortlisting the prospects. Sourcing was amplified with more expansive reach to profiles by taking the advantage of social media.

Present Innovative approaches backed with Technology advancements made possible to sort through more resumes than ever before, and also greatly increased chances of finding someone who's the perfect fit for the role in question. Numerous means to source increased the opportunities to find candidates who were outside the reach of recruiters

Till date, recruiters involved themselves in a tedious process of coordinating with prospects in bringing them for interview, coordinating for interview has become a time-consuming and often resulting in 
frustration for both parties. Spade work of the process is now shall be carried with newly burgeoning interview scheduling software.

These software powered with AI enables recruiters to focus on bringing in right resources at the right time for carrying out selection process, it makes scheduling, organizing and coordination jobs easier and in total, it makes it much easier to promote effectiveness and efficiency in Recruitment process.

While filling the top-tier talent, it is inevitable to identify the most suitable candidature as Job Description and Specifications are more definite in nature and such positions need to be closed without any delay as it may create unrest from both the ends for which recruitment process need to be carried at a quicker pace

It is also important for the recruiting team to be as transparent as possible and it demands for faster communication not only when profile is considered but also even when the candidate was not shortlisted, it shows how organization respects the time of the candidate.

Modern business operations requires human resources with updated skillset. Recruiters will be uncertain about their competencies and learning levels and leaves them grey. New recruitment tools will assist the recruiters to assess the candidates to a larger extent and provide those required inputs for further screening and shortlisting the candidates

AI based data analysis adapted to recruitment process will empower recruiters to pick the profiles more rationally and accurately, saving their time and efforts which results in high rate of success with greater productivity.

\section{Literature Review}

Good number of organizations are using online knowledge management systems to hire employees and availing the advantages of the World Wide Web. These are termed as e-recruitment systems and automate the process of publishing positions and receiving CVs (Faliagka, 2012, p. 523).

E-recruitment systems have seen an explosive expansion in the past few years (De Meo et al., 2007), allowing HR agencies and HR departments to target a very wide audience at a small cost. Several recruitment systems have been proposed with an objective to automate and speed-up the recruitment process, leading to a better overall user experience and increasing efficiency.

Even at present, there has been little research on the impact of e-recruitment on the recruitment process as a whole. Moreover, much of the research tend to focus on the design of corporate recruitment websites (Selden \& Orenstein, 2011).

Moreover, little attention has been given to the impact of technology on the recruitment process as a whole (Parry \& Tyson, 2009).

Despite the apparently widespread use of e-recruitment, however, a gap seems to have emerged between research and practice (García-Izquierdo, Aguinis, \& Ramos-Villagrasa, 2010), possibly because scholars are struggling to keep up with the sheer pace of change (Anderson, 2003).

Personnel recruitment includes practices and activities carried out by an organization for the purpose of identifying, attracting, and influencing the job choices of competent candidates (Ploy Hart, 2006).

Among various HRM activities, recruitment is one of the human resource (HR) functions that has changed dramatically, from traditional paper-based process to digital or electronic recruitment process, commonly referred to as e-recruitment. A popular form of e-recruitment is video recruitment. It is a tool that gathers all the candidates obtained, permitting to systematize the project. However, the most important factor is time saving, which for the business side plays a significant role in planning the recruitment and selection 
strategies. Video recruitment influenced the standard recruitment through the possibility to create and present employers with CVs of job applicants in the form of a video. It is currently a very popular method in Western Europe, and also in Poland, because unconventional approaches are increasingly appreciated ( Puls HR, Video CV 2017).

Video recruitment is to help the human resource management team in less time-consuming but reliable verification of incoming applications. There are two types of video recruitment: an automatic (asynchronous) form and a live form (Kluza, 2015, p. 88). The live form is much more popular because 27 companies have been using it for a long time, mainly involving a tool like Skype. It is associated with a direct interview and few aspects distinguish it from the same. The most important of them is the possibility to organize it without the need to visit a candidate in the recruiter's office. It can take place independent of the place where both parties are staying. Whilst Skype was and is being used to interview candidates, particularly for international recruitment, very few HR teams were familiar with "one-way" video interviews, where candidates record their answers to the questions asked and the employers reviews them at a later time.

Today, the term "video interviewing" is a hot topic amongst many innovations in HR technology and whilst the term encompasses "live" video interview, there is a growing demand for the asynchronous model (Rupert, 2014). It involves building a platform on which incoming applications are collected. Interview questions are generated by the system, the answers are recorded, archived and sent to the person responsible for the selection.

One-way pre-recorded interviews save considerable time and are more convenient. As there is scheduling required, candidates can record their interviews when it suits them - without any disruption to their working day (Rupert, 2014).

Video recruitment is still a controversial and relatively new way of personnel recruitment. Nevertheless, its development is inevitable and probably in the future, it is forecasted that all phases of the selection process will be transferred to the online environment. It is worth emphasizing that the use of this tool does not mean saving time and costs by lowering the efficiency and quality of the recruitment project. On the contrary, it is more transparent, generates wider pool of candidates and helps to select better candidates (Buckley et al. 2004).

Video recruitment has many supporters in Western Europe, is very dynamic in the United States and although it is only taking its first steps in the Polish market, it has great potential to remain a key determinant of most recruitment and selection processes

\section{Objectives of the Study:}

This study envisages the effect of e-recruitment on the design of the recruitment process. The core area of study of this paper is to analyse...

- The Impact of Modern Technologies on Recruitment process as a whole

- The paper attempts to look for the possibilities of including modern technologies in the recruitment and selection strategies of the organization.

- To understand how does AI based technologies help Recruitment and selection strategies to be effective and efficient

- Does this modern approach enables business organizations limiting the length of the recruitment process in terms of time and possibility to decrease the costs? of hiring the right candidate

\section{Methodology}

Content analysis method is adopted to review the literature and subcategories were formed to analyse the research. Literature was collected from 40 articles of a reputed journal from 2000 to 2021. 
Desk based research was made gathering various contributions made by Industry and academia in this area with an objective to explore latest trends in recruitment with special focus on using AI in the recruitment process

The impact of using latest technologies in recruitment process was deeply studied and conclusions were drawn on rational basis. This study can ignite and facilitate further explorations in this field as there is good scope for expansion

\section{AI \& Recruitment Process}

Many organizations now are leveraging AI to improve the efficiency of their employee engagement, recruitment, performance management, workforce management, and succession planning processes, and other processes as well. AI is particularly well-suited for these roles, and it helps HR professionals to focus on complex issues, and leave mundane tasks to be performed by technology.

\subsection{Artificial Intelligence (AI) \& Application Tracking System (ATS )}

AI for recruiting is the application of artificial intelligence to the hiring process, where machine learning will be enabled to pickpotential prospect, as well as automate manual tasks in the recruitment process.

This technology is designed to streamline or automate some part of the recruiting workflow, especially repetitive, high-volume tasks.

For example, software that applies machine learning to resumes to auto-screen candidates or software that conducts sentiment analysis on job descriptions to identify potentially biased language.

AI recruitment software also leverages the wealth of data in your ATS to generate insights into your talent pool. Artificial intelligence can help recruiters with blind screening for reduced bias, as well as visibility into past successful candidates that have remained at the organization

\subsection{Recruitment the AI way:}

5.2.1 Talent acquisition: Automation of processes on multiple fronts is a real game-changer in active recruitment. These can be end-to-end, right from writing the perfect job description (JD) for an open position to even conducting video-based interviews. A poorly-constructed JD that is not attractive may not get the required interest from the right candidates. However, there are several tools that can help you draft the perfect JD. These are usually of two kinds; an online assistant that can help you improve the JD in realtime, while it is being written, or one wherein you can feed an existing JD into a tool that will give you recommendations on how to make the JD more effective.

5.2.2 Resume scouting: Traditionally the recruiter is the person who sources the resume from various sources. This is a time-consuming process, and is also prone to human errors. There are many tools available today that can match your job description to resumes on recruitment portals and help you build a database of the most relevant candidates. These AI-powered tools use pattern matching algorithms to make sure the resume is a close match to that of the job description. They typically use AI and pattern matching algorithms to match resumes to job descriptions.

For example, if the recruiter is looking for a marketing professional with 3-5 years of experience with a salary of Rs 12, 00,000 per annum who stays within $10 \mathrm{~km}$ of their office, the standard search may throw up 30 candidates. However, using AI, the software is capable of suggesting that if the experience desired is increased to 6 years and the salary to Rs 15, 00,000, there would be 50 candidates ideal for the profile. This data is useful to recruiters who need to understand where to get the maximum best-suited candidates from. This results in the elimination of manual efforts and a significant reduction in the number of unsuitable candidates, thus improving the process. 
5.2.3 Video-based interviews: Certain HR-tech solution companies have also come up with video-based interviewing, wherein in-built facial recognition AI technology is used. The software analyses the facial expressions and reaction of the candidate and then gives a report such as whether the candidate was getting prompted by someone else or the candidate appeared to be telling the truth, etc. Such tools also gauge other features like communication skills, body language, and confidence, thus, helping the recruiter make an informed decision.

5.2.4 Multifunctional chatbots: Chatbots are increasingly being utilized for various HR operations ranging from talent acquisition to guaranteeing employee satisfaction. In terms of the recruitment process, these bots not only address basic queries that a potential employee might have, but also collect standard information from candidates about their skills and competencies. Some of them can even carry out primary interviews of candidates and evaluate them based on specific keywords. This is particularly advantageous as it not just saves time but also lets HR professionals conduct the further rounds of interview with better nuanced information about the candidate.

Today, chatbots even conduct interactive employee training sessions, outputs of which have been noticed to be far more interesting than traditional training methods. They can also disburse information regarding detailed company processes, particularly to newer recruits, provide new employees with performance-based feedback instantly, thereby ensuring higher employee satisfaction.

5.2.5 Predictive Analytics: While most HR workspaces conventionally collect large amounts of data, the truth is that it is not utilized to its full potential. AI is adept at swiftly analysing massive amounts of data, identifying patterns and drawing conclusions, much faster and more accurately than manually possible by humans. This can help HR in making recommendations based on predictive analytics, which are highly useful in increasing employee retention rates, rolling out the better selection and evaluation processes, etc.

\subsection{Conclusion}

The changing needs and demands of the industry have forced organizations to adopt these practices and three in five HR leaders believe that if HR functions are not modernized in time, there exists a danger of becoming irrelevant in the sector. Having said that, most HR personnel are still wary of AI-related developments primarily because they believe it requires immense knowledge and technical skills to equip themselves with the necessary expertise needed to incorporate these applications and practices in their daily functioning. There also exists a fear of this automation, ultimately leading to loss of jobs.

HR function being a cost centre in the organizations, the success of HR function lies in bringing down the cost various HR activities with special focus on recruiting and training. Recruiters role not only gets simplified but also enable HR professionals to be more productive as AI makes their jobs easy and cost effective

Recruiters will be able to conduct proactive strategic hiring rather than spend most of their time with reactive back filing

Recruiters will have more time to spend with candidates in-person to build relationships and help determine culture fit.

Recruiters will able to close the loop with hiring managers as AI allows them to use data to show recruiting KPIs including quality of hire.

AI for recruiting is the application of artificial intelligence to the recruitment function that is designed to streamline or automate some part of the recruiting workflow, especially repetitive high-volume tasks.

The main benefits of using AI include saving recruiters' time by automating high-volume tasks and improving quality of hire through standardized job matching. 
The major challenges of using AI for recruiting include requiring a lot of data, the potential to learn human biases, and scepticism of new technology by HR professionals.

The innovations in AI for recruiting are intelligent screening software that automates resume screening, recruiter chatbots that engage candidates in real-time, and digitized interviews that help assess a candidate's fit.

AI will change the recruiter role through augmented intelligence which will allow recruiters to become more proactive in their hiring, help determine a candidate's culture fit, and improve their relationships with hiring managers by using data to measure KPIs such as quality of hire.

However, it is important to remember that AI-powered software and applications cannot entirely replace the human component in the HR process. If anything, they can improve the overall effectiveness and help the industry move one step closer to what is considered to be the future of HR.

\section{References}

[1] Lauren Levine Blogs:(2020)

[2] New Technologies in the Recruitment Process (2018): Celina Sołek-Borowska, Maja Wilczewska

[3] Cornelius, J. König, Eva Jöri., and PatriziaKnüsel. (2011), The Amazing Diversity of Thought: A Qualitative Study on How Human Resource Practitioners Perceive Selection Procedures. Journal of Business and Psychology, Vol. 26, No. 4. pp. 437-452

[4] Dennis Campbell..(2012) .Employee Selection as a Control System. Journal of Accounting Research, Vol. 50, No. 4. pp. 931-966

[5] Don Boyd., Hump Lankford. Susanna Loeb., Matthew Ronfeldt., and JimWyckoff. (2010). The Role of Teacher Quality in Retention and Hiring: Using Applications to Transfer to Uncover Preferences of Teachers and Schools. Journal of Policy Analysis and Management, Vol. 30, No. 1. pp. 88-110

[6] Dr. DilipAher., and Dr. Ghanshyam D, Giri. (2018). A study of Recruitment and Selection process with Special reference to manufacturing industries in Pimpri- chinchwadmidc. Elk Asia pacific journal of human resource management and organizational behavior, Volume 4, Issue 1

[7] Eunmi Mun. (2010). Sex Typing of Jobs in Hiring: Evidence from Japan. Oxford University Press, Vol. 88, Issues: 5, pp. 1999-2026

[8] Helen D, Arnold, (2013). The Affordable Care Act and International Recruitment and Migration of Nursing Professionals .Indiana Journal of Global Legal Studies, Vol. 20, No. 2 .pp. 1373-1391

[9] Jasjit Singh., and Ajay Agrawal. (2011). Recruiting for Ideas: How Firms Exploit the Prior Inventions of New Hires. Management Science, Vol. 57, No. 1, pp. 129-150

[10] Jed DeVaro., and Hodaka Morita. (2013).Internal Promotion and External Recruitment: a Theoretical and Empirical Analysis. Journal of Labor Economics, Vol. 31, No. 2. pp. 227-269

[11] Katherine Calogero. (2011). become a fan of Government Procurement on Facebook: How the Federal government's acquisition workforce can use social networking websites to recruit New Employees. Public Contract Law Journal, Vol. 40, No. 3. pp. 807-828

[12] Lauren a, River. (2012), Diversity within Reach: Recruitment versus Hiring in Elite Firms. The Annals of the American Academy of Political and Social Science, Vol. 639, pp. 71-90

[13] Leonard Bright., and Cole Blease Graham, Jr. (2015). Why Does Interest in Government Careers Decline among PublicAffairs Graduate Students? .Journal of Public Affairs Education, Vol. 21, No. 4. pp. 575-594

[14] Lieselotte Blommaert., Marcel Coenders., and Frank van Tubergen. (2014), Discrimination of ArabicNamed Applicants in the Netherlands: An Internet-Based Field Experiment Examining Different Phases in Online Recruitment Procedures. Social Forces, Vol. 92, No. 3 ,pp. 957-982

[15] Linda Colley.(2011).The Passing of Youth: How Removal of Traditional Youth Recruitment Policies Contributed to the Ageing of Public Service Workforces. Labour History, No. 101.pp. 177-193 
[16] María Fernanda García, Richard, A. Posthuma and Manuel Quiñones. (2010). How Benefit Information and Demographics Influence Employee Recruiting in Mexico. Journal of Business and Psychology, Vol. 25, No. 3 ,pp. 523-531

[17] Matthew Bidwell. (2011). Paying More to Get Less: The Effects of External Hiring versus Internal Mobility. Administrative Science Quarterly, Vol. 56, No. 3. pp. 369-407

[18] Melody E, Valdini., and Christopher Shortell. (2016).Women's Representation in the Highest Court: A Comparative Analysis of the Appointment of Female Justices. Political Research Quarterly, Vol. 69, No. 4. pp. 865-876

[19] Mir Mohammed Nurul Absar.(2012). Recruitment \& Selection Practices in Manufacturing Firms in Bangladesh. Indian Journal of Industrial Relations, Vol. 47, No. 3 .pp. 436-449

[20] Paul T, Knudson. (2012). Regional Industrial Recruitment in Upstate New York. State \& Local Government Review, Vol. 44, No. 1.pp. 21-32

[21] Samita Sen. (2010). Commercial recruiting and Informal Intermediation: debate over the sardari system in Assam tea plantations, 1860-1900.Modern Asian Studies, Vol. 44, No. 1, pp. 3-28

[22] Shamima Ahmed and Allison Adams. (June 2010). Web Recruiting in Government Organizations: A Case Study of the Northern Kentucky/Greater Cincinnati Metropolitan Region. Public Performance \& Management Review, Vol. 33, No. 4. pp. 653-670 